\title{
Molecular Adsorption Bond Lengths at Metal Oxide Surfaces: Failure of Current Theoretical Methods
}

\author{
J.-T. Hoeft, M. Kittel, M. Polcik, and S. Bao \\ Fritz-Haber-Institut der Max-Planck-Gesellschaft, Faradayweg 4-6, D14195 Berlin, Germany \\ R.L. Toomes, J.-H. Kang, and D.P. Woodruff* \\ Physics Department, University of Warwick, Coventry CV4 7AL, United Kingdom
}

\author{
M. Pascal and C.L. A. Lamont \\ Centre for Applied Catalysis, Department of Chemical \& Biological Sciences, University of Huddersfield, \\ Queensgate, Huddersfield HD1 3DH, United Kingdom \\ (Received 6 February 2001; published 1 August 2001)
}

\begin{abstract}
New experimental structure determinations for molecular adsorbates on $\mathrm{NiO}(100)$ reveal much shorter $\mathrm{Ni}-\mathrm{C}$ and $\mathrm{Ni}-\mathrm{N}$ bond lengths for adsorbed $\mathrm{CO}$ and $\mathrm{NH}_{3}$ as well as $\mathrm{NO}(2.07,1.88,2.07 \AA)$ than previously computed theoretical values, with discrepancies up to $0.79 \AA$, highlighting a major weakness of current theoretical descriptions of oxide-molecule bonding. Comparisons with experimentally determined bond lengths of the same species adsorbed atop $\mathrm{Ni}$ on metallic Ni(111) show values on the oxide surface that are consistently larger $(0.1-0.3 \AA)$ than on the metal, indicating somewhat weaker bonding.
\end{abstract}

DOI: 10.1103/PhysRevLett.87.086101

PACS numbers: 68.43.Fg, 68.47.Gh, 68.49.Jk

Very substantial advances in computational density functional theory (DFT) during the past few years have shown that adsorbate bonding on metal surfaces can be described very precisely, achieving agreement with experimental results in local structural parameters within a few hundredths of an angstrom unit. There is now increasing interest in oxide surfaces, which are widely recognized as being of great importance in applications such as heterogeneous catalysis, but the state of knowledge concerning these surfaces is far more limited. In particular, there is a dearth of experimental structural data [1], especially for adsorbates on oxides, and theory-experiment comparison has had to concentrate on the molecular bonding energy (which is compared with the experimental thermal desorption energy) and the vibrational energies, especially of internal adsorbate vibrations, such as the $\mathrm{C}-\mathrm{O}$ stretching frequency of adsorbed $\mathrm{CO}$ [2]. In an attempt to redress this balance we have performed quantitative structure determinations of the model systems of $\mathrm{CO}, \mathrm{NO}$, and $\mathrm{NH}_{3}$ adsorbed on $\mathrm{NiO}(100)$. Our results show that the current theoretically derived adsorbate-substrate bond lengths on this surface are very seriously in error (by up to $0.79 \AA$ ), and they provide the first quantitative reference data against which future theoretical developments may be evaluated. In an attempt to identify some systematics of the bonding at $\mathrm{NiO}(100)$ based only on experimental structural information, we compare these bond lengths with those measured by the same experimental methods, in the same singly coordinated adsorption sites, on metallic $\mathrm{Ni}(111)$. Our results are consistent with adsorption on the oxide surface being significantly weaker than on the metal, but being much less weak than indicated by current theoretical treatments.
Our experiments use the technique of scanned-energy mode photoelectron diffraction $(\mathrm{PhD})$ [3] which exploits the coherent interference between the directly emitted component of the photoelectron wave field from an adsorbate core level and other components of this wave field elastically scattered by nearby atoms in the substrate. By measuring the changes in the photoemission signal as a function of photon energy (and thus photoelectron kinetic energy and wavelength), modulations associated with specific scattering path lengths switching in and out of phase are observed. Multiple scattering simulations of this process for a series of model trial structures allow the local adsorbate structural environment to be determined. We have established the methodology of this technique [3] and used it to determine the structure of more than 60 adsorbate systems on metal and semiconductor surfaces. Using this same approach we have recently [4] used $\mathrm{N} 1 s \mathrm{PhD}$ to determine the adsorption geometry of $\mathrm{NO}$ adsorbed on a thin $\mathrm{NiO}(100)$ film grown in situ on $\mathrm{Ni}(100)$ using the approach of Kuhlenbeck et al. [5] who characterized the adsorption characteristics of these films in detail and showed that their behavior is very similar to those of cleaved bulk $\mathrm{NiO}$ surfaces. We have now made new $\mathrm{C} 1 s \mathrm{PhD}$ measurements of adsorbed $\mathrm{CO}$ and $\mathrm{N} 1 s$ $\mathrm{PhD}$ measurements of adsorbed $\mathrm{NH}_{3}$ on $\mathrm{NiO}(100)$ thin films prepared in the same way. The experiments were conducted using the BESSY synchrotron radiation source in Berlin on a purpose-built beam line and surface-science end station described elsewhere (e.g., Ref. [4]). The general method of obtaining $\mathrm{PhD}$ modulation spectra from individual $1 s$ photoelectron energy distribution curves recorded at $2 \mathrm{eV}$ steps in photon energy was also described previously [3]. Measurements were made for 


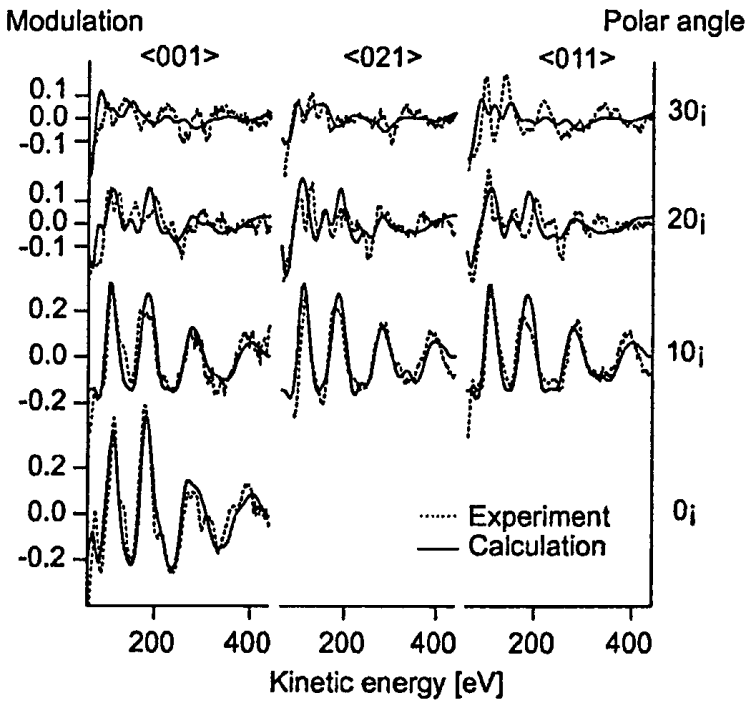

FIG. 1. Comparison of experimental (dashed lines) C $1 s \mathrm{PhD}$ modulation spectra from $\mathrm{CO}$ adsorbed on $\mathrm{NiO}(100)$ recorded at four different polar emission angles in three different azimuths with the results of multiple scattering simulations (solid lines) for the best-fit structure.

$\mathrm{CO}$ adsorbed at temperatures of $60-90 \mathrm{~K}$, and for $\mathrm{NH}_{3}$ adsorbed at $140 \mathrm{~K}$. A representative data set of $\mathrm{PhD}$ spectra recorded in ten different emission directions for the $\mathrm{NiO}(100) / \mathrm{CO}$ system is shown in Fig. 1. Strong modulations with a single period are characteristic of a $180^{\circ}$ scattering geometry from a near neighbor [3], so the fact that these data show such modulations at normal emission, and that the amplitude decays with increasing polar emission angle, is characteristic of an atop adsorption geometry. Multiple scattering simulations [3] confirm this conclusion, and after structural optimization we obtain the best-fit curves of Fig. 1. The optimized geometry gave a $\mathrm{PhD}$ reliability $(R)$ factor [6] value of 0.19 for the set of ten spectra, indicating a good quality fit, and corresponds to $\mathrm{CO}$ adsorbed atop a surface $\mathrm{Ni}$ atom via the $\mathrm{C}$ atom with the $\mathrm{C}-\mathrm{O}$ axis approximately perpendicular to the surface. Tests on models based on different adsorption sites and geometries (including $\mathrm{CO}$ bonded through the $\mathrm{O}$ atom and adsorption atop $\mathrm{O}$ atoms in the $\mathrm{NiO}$ surface) led to far higher $R$-factor values $(>0.4)$ and can clearly be excluded. The adsorbed $\mathrm{NH}_{3}$ molecules were also found to occupy sites atop a surface Ni atom. Table I summarizes the structural parameter values found for $\mathrm{CO}$

TABLE I. Optimum values of the structural parameters forund in this experimental study of $\mathrm{NiO}(100) / \mathrm{CO}$ and $\mathrm{NiO}(100) / \mathrm{NH}_{3}$.

\begin{tabular}{ccc}
\hline \hline Adsorbate & $\mathrm{CO}$ & $\mathrm{NH}_{3}$ \\
\hline$d_{\mathrm{Ni}-\mathrm{C}}, d_{\mathrm{Ni}-\mathrm{N}}$ & $2.07 \pm 0.02 \AA$ & $2.06 \pm 0.02 \AA$ \\
$d_{\mathrm{O}-\mathrm{C}}, d_{\mathrm{O}-\mathrm{N}}$ & $1.15(+0.10 /-0.08) \AA$ & $\cdots$ \\
$\theta_{\mathrm{Ni}-\mathrm{C}}, \theta_{\mathrm{Ni}-\mathrm{N}}$ & $7(+5 /-3)^{\circ}$ & $7 \pm 6^{\circ}$ \\
$\theta_{\mathrm{O}-\mathrm{C}}, \theta_{\mathrm{O}-\mathrm{N}}$ & $12 \pm 12^{\circ}$ & $\ldots$ \\
$\Delta z_{\mathrm{Ni}}$ & $0.03(+0.06 /-0.08) \AA$ & $0.11 \pm 0.02 \AA$ \\
\hline \hline
\end{tabular}

and $\mathrm{NH}_{3}$ adsorption; for $\mathrm{CO}$ adsorption the local site and molecular orientation (Fig. 2) are consistent with previous theoretical treatments $[7,8]$. Notice that any local expansion of the outermost $\mathrm{Ni}$ atom layer $\left(\Delta z_{\mathrm{Ni}}\right)$ is either negligible or very small, as are any tilts of the Ni-N, Ni-C, or $\mathrm{C}-\mathrm{O}$ bonds ( $\theta$ parameters).

While the local adsorption site for $\mathrm{CO}$ (and for NO) is in good qualitative agreement with the results of the previous theoretical studies $[5,7,8]$, the quantitative values of the local Ni-molecule bond lengths are in very poor agreement. These are shown for all three adsorbate systems in Table II. The theoretical Ni-N bond length for NO adsorption [5] is $0.22 \AA$ longer than the experimental value, while the Ni-C distances found in the two theoretical treatments are $0.42 \AA$ [7] and $0.79 \AA$ [8] longer than the experimental value. Differences of this scale indicate gross errors in the description of the bonding by the theoretical calculations. Another manifestation of this misdescription, which is already recognized, is the bonding energy of $\mathrm{CO}$ and $\mathrm{NO}$ on $\mathrm{NiO}(100)$; the theoretical calculations yield values for $\mathrm{CO}$ of $0.25 \mathrm{eV}$ [7] and $0.08 \mathrm{eV}$ [8] and for $\mathrm{NO}$ of $0.17 \mathrm{eV}$ [5], while the corresponding experimental values from thermal desorption are 0.30 and $0.57 \mathrm{eV}$ [9].

The general problem of accurately describing oxidemolecule bonding has been the subject of recent debates [10-12]. In the case of $\mathrm{MgO}(100) / \mathrm{CO}$, standard local density approximation calculations significantly underestimate the adsorption energy of this very weak adsorption system, and while stronger bonding occurs if one does not include generalized gradient correction [11] this solution is not generally regarded as acceptable [10]. The case of $\mathrm{NiO}$ is potentially even more difficult, because standard DFT methods fail to give a proper description of the electronic structure, including the band gap, and in particular do not properly describe the occupation of the Ni $3 d$ states [13]; as such they are ill-suited to describe adsorbate bonding. Potentially more fruitful for $\mathrm{NiO}$ adsorption are the SCF (self-consistent field) methods, including various correction terms and configuration interaction. However, while the citations given here for this type of calculation on $\mathrm{NiO}$

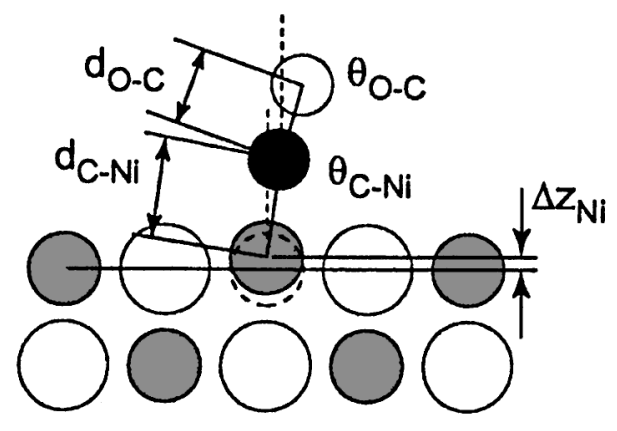

FIG. 2. Schematic side view of the best-fit adsorption geometry of $\mathrm{CO}$ on $\mathrm{NiO}(100)$, including a definition of the structural parameters given in Table I. The shaded and open circles correspond to $\mathrm{Ni}$ and $\mathrm{O}$ atoms, respectively. 
TABLE II. Comparison of Ni-C and Ni-N nearest neighbor distances obtained from $\mathrm{PhD}$ studies of $\mathrm{CO}, \mathrm{NO}$, and $\mathrm{NH}_{3}$ adsorption on $\mathrm{NiO}(100)$ and $\mathrm{Ni}$ surfaces, and of theoretical values of the same parameters for $\mathrm{CO}$ and $\mathrm{NO}$ adsorption on $\mathrm{NiO}(100)$.

\begin{tabular}{lccc}
\hline \hline \multicolumn{1}{c}{ Substrate } & $d_{\mathrm{Ni}-\mathrm{C}}(\mathrm{CO}) / \AA$ & $d_{\mathrm{Ni}-\mathrm{N}}(\mathrm{NO}) / \AA$ & $d_{\mathrm{Ni}-\mathrm{N}}\left(\mathrm{NH}_{3}\right) / \AA$ \\
\hline $\mathrm{NiO}(100)$ & $2.07 \pm 0.02$ & $1.88 \pm 0.02$ & $2.06 \pm 0.02$ \\
$\mathrm{NiO}(100)$ theory & $2.49[7], 2.86[8]$ & $2.1[5]$ & $\cdots$ \\
$\mathrm{Ni}(111)$ atop & $1.77 \pm 0.02[15]$ & $\cdots$ & $1.97 \pm 0.03[16]$ \\
& $($ coadsorbed with $\mathrm{O})$ & $1.84 \pm 0.07[18]$ & $\ldots$ \\
$\mathrm{Ni}(111)$ hollow & $1.93 \pm 0.03[17]$ &
\end{tabular}

are for work which is some ten years old, very recent attempts to reinvestigate this situation with the latest methods have so far failed to yield any improvement [12]. One possible underlying problem is the use of small clusters in these calculations, which will fail to describe the electronic band properties, and may not be properly converged. In this regard we note that the other SCF calculations of this type showed that $\mathrm{CO}$ and $\mathrm{NO}$ bonding strengths on $\mathrm{NiO}$ could be increased by using a "weak potential," effectively lowering the ionicity [14]. This idea can only be evaluated effectively, however, through the use of larger clusters to eliminate the effects of the assumed charge distribution.

While it is not possible to infer the details of bonding from measured bond length alone, the values of this parameter are expected to follow some general trends with bonding character. Table II includes a comparison of the local $\mathrm{Ni}-\mathrm{C}$ and $\mathrm{Ni}-\mathrm{N}$ bond lengths for adsorption of the three species on the $\mathrm{NiO}(100)$ surface and on metallic $\mathrm{Ni}(111)$ (the only Ni surface on which the local structure of all three species have been studied) [15-18]. In constructing this table we used only values for the metal surfaces obtained by the same $\mathrm{PhD}$ technique, as this ensures that nay systematic errors of the method should be common to all measurements. Note, however, that for those systems investigated by other methods [notably $\mathrm{Ni}(111) / \mathrm{CO}$ and $\mathrm{Ni}(111) / \mathrm{NO}$ by low-energy electron diffraction [1]] there is excellent quantitative agreement between the methods. One complication in making these comparisons is that a chemical bond length depends not only on the character of the bonding but also on the coordination [19]. All three molecules are singly coordinated in atop sites on $\mathrm{NiO}(100)$, so ideally one should compare these bond lengths with similar atop site adsorption on metallic $\mathrm{Ni}$. For $\mathrm{NH}_{3}$ adsorption on $\mathrm{Ni}(111)$, atop site adsorption is standard. On clean $\mathrm{Ni}(111)$ both $\mathrm{CO}$ and $\mathrm{NO}$ occupy threefold coordinated hollow sites at all coverages studied up to 0.5 monolayer, and the bond lengths corresponding to these sites are also given in Table II. In the case of CO adsorption, however, preadsorption of atomic $\mathrm{O}$ leads to the $\mathrm{CO}$ occupying atop sites, and the bond length for this coadsorption situation is given in the table. Clearly the presence of the coadsorbed $\mathrm{O}$ may influence the character of the Ni-CO bonding, but the reduction in $\mathrm{Ni}-\mathrm{CO}$ bond length in the singly coordinated site relative to that in the threefold coordinated site does follow the expected trend for the coordination change [19]. This singly coordinated Ni-C bond length on $\mathrm{Ni}(111)$ of $1.77 \AA$ is also similar to that found for atop adsorption on $\mathrm{Ni}(100)$ [1]. For $\mathrm{CO}$ and $\mathrm{NH}_{3}$ adsorption the table shows a clear trend for $\mathrm{Ni}-\mathrm{C}$ and $\mathrm{Ni}-\mathrm{N}$ distances to be longer on the $\mathrm{NiO}$ surface by approximately $0.1-0.3 \AA$.

The situation is less clear for the case of NO because on $\mathrm{Ni}(111)$ this species has only been found in the threefold coordinated site, and the difference in bond lengths shown in the table between $\mathrm{NiO}(100)$ and $\mathrm{Ni}(111)$ is smaller than the estimated precision. Although quantitative structural information is available for singly coordinated NO adsorption on $\mathrm{Ni}(111)$, the results shown in Table II for $\mathrm{CO}$ adsorption in the two different coordinations certainly suggest that we might expect atop NO on metallic Ni to have a Ni-N distance shorter than the value of $1.84 \AA$ found for the threefold coordinated site. There is also some relevant comparative data for $\mathrm{NO}$ for the case of a saturated $(2 \times 2)$ layer of $\mathrm{NO}$ on $\mathrm{Ru}(0001)$ in which atop and threefold coordinated hollows are both occupied; in this system the Ru-C bond lengths are 1.72 and $2.08 \AA$ for single and threefold coordination, respectively [20]. This clearly reinforces the view that the Ni-N bond length found for $\mathrm{NO}$ adsorbed on $\mathrm{NiO}(100)$ is significantly longer than that which would correspond to NO in a singly coordinated bond on metallic Ni.

The fact that the local bond lengths are shorter for $\mathrm{CO}$, $\mathrm{NO}$, and $\mathrm{NH}_{3}$ adsorption on $\mathrm{Ni}(111)$, relative to adsorption on $\mathrm{NiO}(100)$, is consistent with the higher desorption temperature and higher implied bonding energies on the metal surface. Nevertheless, the adsorption bond lengths measured on $\mathrm{NiO}(100)$ are sufficiently short to indicate true bond formation, and not simply electrostatic interaction as has been concluded for $\mathrm{CO}$ adsorption on $\mathrm{NiO}(100)$ on the basis of current theoretical treatments. Indeed, while the shorter Ni-molecule bond lengths obtained on metallic $\mathrm{Ni}$ surfaces are generally consistent with expectations for covalent bonding, the bond lengths on $\mathrm{NiO}$ are very much smaller than those which may be estimated from a sum of van der Waals radii [21] of 3.18 and $3.33 \AA$ for $\mathrm{Ni}-\mathrm{N}$ and $\mathrm{Ni}-\mathrm{C}$, respectively. Clearly there is a need for further theoretical work, but our results provide the first quantitative structural information by which the results of these calculations may be judged.

This work was supported by the German Federal Ministry of Education, Science, Research and Technology 
(Contract No. 05 625EBA 6), by the Fonds der Chemischen Industrie, by the European Community through Large Scale Facilities support to BESSY, and by the Physical Sciences and Engineering Research Council (UK). The authors are also pleased to acknowledge valuable discussions with Lars Pettersson and Nic Harrison.

*Also at Fritz-Haber-Institut der Max-Planck-Gesellschaft, Faradayweg 4-6, D14195 Berlin, Germany

[1] P. R. Watson, M. A. Van Hove, and K. Hermann, NIST Surface Structure Database Version 3.0, NIST Standard Reference Data Program, Gaithersburg, MD, 1999.

[2] See, e.g., G. Pacchioni, Surf. Rev. Lett. 7, 277 (2000).

[3] D. P. Woodruff and A. M. Bradshaw, Rep. Prog. Phys. 57, 1024 (1994).

[4] R. Lindsay, P. Baumgartel, R. Terborg, O. Schaff, A. M. Bradshaw, and D. P. Woodruff, Surf. Sci. 425, L401 (1999).

[5] H. Kuhlenbeck, G. Odörfer, R. Jaeger, G. Illing, M. Menges, Th. Mull, H.-J. Freund, M. Pöhlchen, V. Staemmler, S. Witzel, C. Scharfschwerdt, K. Wennemann, T. Liedtke, and M. Naumann, Phys. Rev. B 43, 1969 (1991).

[6] R. Dippel, K-U. Weiss, K-M. Schindler, P. Gardner, V. Fritzsche, A. M. Bradshaw, M. C. Asensio, X. M. Hu, D. P. Woodruff, and A.R. Gonzalez-Elipe, Chem. Phys. Lett. 199, 625 (1992).

[7] G. Pacchioni, G. Cogliandro, and P. S. Bagus, Surf. Sci. 255, 344 (1991).
[8] M. Pöhlchen and V. Staemmler, J. Chem. Phys. 97, 2583 (1992).

[9] R. Wichtendahl, M. Rodriguez-Rodrigo, U. Härtel, H. Kuhlenbeck, and H.-J. Freund, Surf. Sci. 423, 90 (1999).

[10] F. Illas, G. Pacchioni, A. Pelmenschikov, L. G. M. Pettersson, R. Dovesci, C. Pisani, K. M. Neyman, and N. Rösch, Chem. Phys. Lett. 306, 202 (1999).

[11] R. Wu and Q. Zhang, Chem. Phys. Lett. 306, 205 (1999).

[12] L. G. Pettersson, Faraday Discuss. 114, 229 (1999).

[13] See, e.g., N. M. Harrison, V. R. Saunders, R. Dovesi, and W. C. Mackrodt, Philos. Trans. R. Soc. London A 356, 75 (1998).

[14] M. A. Nygren and L. G. M. Pettersson, J. Electron Spectrosc. Relat. Phenom. 69, 43 (1994).

[15] J.-H. Kang, R. L. Toomes, J. Robinson, D. P. Woodruff, R. Terborg, M. Polcik, J. T. Hoeft, P. Baumgärtel, and A. M. Bradshaw, J. Phys. Chem. B 105, 3701 (2001).

[16] K-M. Schindler, V. Fritzsche, M. C. Asensio, P. Gardner, D. E. Ricken, A. W. Robinson, A. M. Bradshaw, D. P. Woodruff, J. C. Conesa, and A. R. Gonzalez-Elipe, Phys. Rev. B 46, 4836 (1992).

[17] M.E. Davila, M.C. Asensio, D. P. Woodruff, K-M. Schindler, Ph. Hofmann, K-U. Weiss, R. Dippel, P. Gardner, V. Fritzsche, A. M. Bradshaw, J. C. Conesa, and A. R. González-Elipe, Surf. Sci. 311, 337 (1994).

[18] R. Lindsay, A. Theobald, T. Gießel, O. Schaff, A. M. Bradshaw, N. A. Booth, and D. P. Woodruff, Surf. Sci. 405, L566 (1998).

[19] L. Pauling, The Nature of the Chemical Bond (Cornell University Press, Ithaca, 1960), 3rd ed.

[20] M. Stichler and D. Menzel, Surf. Sci. 391, 47 (1997).

[21] A. Bondi, J. Phys. Chem. 68, 441 (1964). 